\title{
On an improved computational solution for the 3D HCIR PDE in finance
}

\author{
Fazlollah Soleymani, Ali Akgül, Esra Karatas Akgül
}

\begin{abstract}
The aim of this work is to tackle the three-dimensional (3D) HestonCox-Ingersoll-Ross (HCIR) time-dependent partial differential equation (PDE) computationally by employing a non-uniform discretization and gathering the finite difference (FD) weighting coefficients into differentiation matrices. In fact, a non-uniform discretization of the 3D computational domain is employed to achieve the second-order of accuracy for all the spatial variables. It is contributed that under what conditions the proposed procedure is stable. This stability bound is novel in literature for solving this model. Several financial experiments are worked out along with computation of the hedging quantities Delta and Gamma.
\end{abstract}

\section{Introduction and motivation}

In option pricing, basically a set of stochastic differential equations (SDEs) corresponding to spatial variables, e.g., stock, rate of interest, and volatility, is taken into account for modeling derivative products, [14]. Accordingly, the option pricing for derivatives can be tackled via this set of SDEs or via the corresponding PDE formulations. On the other hand, since analytical solutions for such models are not mainly known, computational methods by virtue of simulations or discretizations must be taken into consideration, see

Key Words: Financial option pricing; stochastic interest rate; Heston model; nonuniform finite difference method; quadratically convergent.

2010 Mathematics Subject Classification: Primary 91B26; Secondary 91B25.

Received: 23.10.2018

Accepted: 31.01 .2019 
e.g. $[10,18,22,24]$. For some discussions concerning the problem of pricing option, an interested reader may refer to the work [5].

One improvement of the Black-Scholes model was discussed by Heston in [12] by considering the stochastic volatility. Recently, the Heston's stochastic volatility problem was thoroughly applied in the field of reinsurance, for instance refer to [32].

The presence of foreign exchange products which all have a long lifetime as well as so sensitive to implied volatilities or skews in the market, make it necessary and requisite to study and develop revised models with stochastic interest rates, like the Heston-Cox-Ingersoll-Ross (HCIR) problem or the Power-Reverse Dual-Currency (for more, refer to [26] and the references therein).

The classical Cox-Ingersoll-Ross (CIR) process for non-negative stochastic variable $R(\tau)$ was introduced in [4] as comes next:

$$
\begin{aligned}
d R(\tau) & =\kappa(\eta-R(\tau)) d \tau+\theta \sqrt{R(\tau)} d W(\tau), \\
R(0) & =R_{0}
\end{aligned}
$$

wherein $W(\tau)$ is the standard process of Wiener and $\kappa, \eta, \theta$ are the speed of adjustment, the mean and the volatility, respectively.

It is requisite to develop and consider interest rates to be stochastic to have a better outlook of the option price in market, see e.g., $[1,7,16]$. The HCIR hybrid model was discussed in [9] by proposing approximations based on the characteristic functions. Actually, the resulting method for pricing takes the merit of quicker evaluations for characteristic function.

The HCIR model [9] as a system of three SDEs is given by:

$$
\begin{aligned}
d S(\tau)= & R(\tau) S(\tau) d \tau+S(\tau) \sqrt{V(\tau)} d W^{(1)}(\tau), \\
d V(\tau)= & \kappa(\eta-V(\tau)) d \tau+\sigma_{1} \sqrt{V(\tau)} d W^{(2)}(\tau), \\
d R(\tau)= & a(b(\tau)-R(\tau)) d \tau+\sigma_{2} \sqrt{R(\tau)} d W^{(3)}(\tau), \\
& 0<\tau \leq T, \quad T>0,
\end{aligned}
$$

where $S(\tau), V(\tau)$, and $R(\tau)$ stand for the asset price, its variance and rate of interest at $\tau$, respectively. Furthermore, $b>0$ is a function in terms of time. And, it should match the current term structure of the interest rates. This consideration is also an extension over (1), at which the function $b$ was considered to be constant.

Here the parameters $\kappa, \eta, \sigma_{1}, \sigma_{2}, a$ are positive parameters, while $W^{(1)}(\tau)$, $W^{(2)}(\tau), W^{(3)}(\tau)$ are the three continuous independent Wiener processes. The 
correlation parameters are $-1 \leq \rho_{12}, \rho_{13}, \rho_{23} \leq 1$, and form the following symmetric positive semi-definite correlation matrix:

$$
M=\left(\begin{array}{ccc}
1 & \rho_{12} & \rho_{13} \\
* & 1 & \rho_{23} \\
* & * & 1
\end{array}\right) .
$$

As discussed in [9], the fair pricing procedure should be carried out by computational schemes since the corresponding high-dimensional PDEs, constructed for such options, do not admit any analytical or semi-analytical solutions.

In [11], the author investigated how a non-uniform discretization can be applied for pricing the HCIR PDE. In fact, the convergence speed of their estimations is only 1 for the second derivative and 2 for the first derivative terms, though their FD approximations have second-order truncation error, [20]. This motivates us to focus on solving this problem efficiently.

The contributions of this work are organized as comes next.

- Considering a non-uniform discretization using a special grid with a focus on the important area for option pricing under the HCIR PDE. To this end, the 3D time-dependent linear PDE is semi-discretized (refer to [21]) along the state variables using a non-uniform structure of the points via a completely second-order finite difference (FD) formulas.

- Toward this goal, we attain a system of coupled ODEs under an initial condition, which could efficiently be computed via the time-marching methods. It is proposed that the presented numerical scheme is quadratically convergent in space and time.

- We contribute that the new method is stable under several conditions. This is also novel in contrast to the existing papers for this model, see for example [11].

- Studying the convergence of our non-uniform (adaptive) FD method is another contribution of this work.

The remaining parts of this article are summarized as comes next. In Section 2, the Heston stochastic volatility problem alongside stochastic interest rate for option pricing is introduced as a $3 \mathrm{D}$ time-varying 2 nd-order $\mathrm{PDE}$ subject to (non-smooth) payoffs. Section 3 presents a (totally) second-order FD procedure for semi-discretization of the PDE.

Afterward in Section 4, the method-of-lines technique is considered to build a set of ODEs with time-varying system matrix. All the side conditions are imposed therein as well. 
Thence, a method to march along time for the set of ODEs is provided in Section 5 and it is analytically illustrated that the presented numerical procedure is conditionally time-stable when $b$ is not changing by time.

We illustrate the efficiency and stability of the new scheme in Section 6 . It is noted that Greeks, i.e., the hedging quantities are significant in practice and the proposed scheme should furnish the option price and these hedging quantities to be not only accurate but also to be stable.

In fact, each Greek contributes to a various dimension of risk and the objective of a trader is to handle the Greeks such that all risks are taken into account. Here the Greeks, Gamma and Delta, which are defined as follows:

$$
\Delta=\frac{\partial u}{\partial s}, \quad \Gamma=\frac{\partial^{2} u}{\partial s^{2}}, \quad u(s, v, r, t) \text { is the pricing function, }
$$

are computed using the proposed scheme. Lastly, in Section 7, several conclusions are provided.

\section{PDE-based problem via Heston model with stochastic interest rate}

The PDE problem associated with the SDE model (2) for fair values of Europeantype options is given as a convection-diffusion-reaction (CDR) PDE [9] in what follows:

$$
\begin{aligned}
\frac{\partial u(s, v, r, t)}{\partial t}= & \frac{1}{2} s^{2} v \frac{\partial^{2} u(s, v, r, t)}{\partial s^{2}}+\frac{1}{2} \sigma_{1}^{2} v \frac{\partial^{2} u(s, v, r, t)}{\partial v^{2}}+\frac{1}{2} \sigma_{2}^{2} r \frac{\partial^{2} u(s, v, r, t)}{\partial r^{2}} \\
& +\rho_{12} \sigma_{1} s v \frac{\partial^{2} u(s, v, r, t)}{\partial s \partial v}+\rho_{13} \sigma_{2} s \sqrt{v r} \frac{\partial^{2} u(s, v, r, t)}{\partial s \partial r} \\
& +\rho_{23} \sigma_{1} \sigma_{2} \sqrt{v r} \frac{\partial^{2} u(s, v, r, t)}{\partial v \partial r} \\
& +r s \frac{\partial u(s, v, r, t)}{\partial s}+\kappa(\eta-v) \frac{\partial u(s, v, r, t)}{\partial v} \\
& +a(b(T-t)-r) \frac{\partial u(s, v, r, t)}{\partial r}-r u(s, v, r, t),
\end{aligned}
$$

wherein $\tau=T-t$.

Here, the test of Feller is often taken into account to be satisfied. For the process of variance, this criterion is given by

$$
2 \kappa \eta>\sigma_{1}^{2},
$$


and for the process of interest rate, it is as comes next:

$$
2 a b(\tau)>\sigma_{2}^{2}, \quad \forall 0 \leq \tau \leq T .
$$

Here a merit of the discussed method in this work is that it does not demand for the criteria (6) and (7) to be satisfied in order to give an accurate numerical solution.

For call and put options, the payoffs are provided as follows:

$$
u(s, v, r, 0)=(s-E)^{+},
$$

and

$$
u(s, v, r, 0)=(E-s)^{+},
$$

wherein $E$ is the price of strike, respectively.

The side conditions (for a call option) along $s$ are defined by:

$$
\begin{aligned}
u(s, v, r, t) & =0, & s & =0, \\
\frac{\partial u}{\partial s}(s, v, r, t) & =1, & s & =s_{\max } .
\end{aligned}
$$

See also [17] for some discussions about alternative choices of the boundary conditions. Noting that at the boundary $v=v_{\max }$, the following condition of Dirichlet is imposed:

$$
u(s, v, r, t)=s, \quad v=v_{\max } .
$$

The important point is that the HCIR PDE at the boundary $v \downarrow 0$ is degenerate, and thus no boundary is prescribed. Therefore, we take into account that the discretized PDE at these boundary nodes holds true, see the spirit of such a consideration in [6]. Recalling again that for these boundaries, we do not impose the reduced PDE (although many of the 2 nd order derivative terms in (5) vanish once $v, r \downarrow 0)$.

The system of SDEs (2) for $v$ and $r$ in (2) are of the same type and, thus, also the 1st- and 2nd-order derivatives $\frac{\partial u}{\partial r}, \frac{\partial^{2} u}{\partial r^{2}}$ and their coefficients in the PDE (5) are of the same form as for $v$. Therefore, at $r \downarrow 0$, the HCIR model has degeneracy and no side condition is prescribed. Therefore, the obtained equations at such boundaries are taken into account for the boundary nodes.

Once $r=r_{\max }$, the boundary condition of Neumann type is imposed:

$$
\frac{\partial u}{\partial r}(s, v, r, t)=0, \quad r=r_{\max }
$$

Similar relations could be defined for the put-type options. 
Generally speaking, the PDE (5) is given in an unbounded domain, which is $(s, v, r, t) \in \Omega \times(0, T]$, where $\Omega=[0,+\infty) \times[0,+\infty) \times[0,+\infty)$. To deal with this numerically, we must localize it into a numerical truncated domain ([19]). Because, the problem is well-posed and a solution exist [2], the numerical domain could be taken into account to be bounded, for example,

$$
\Omega=\left[0, s_{\max }\right] \times\left[0, v_{\max }\right] \times\left[0, r_{\max }\right]
$$

wherein $s_{\max }, v_{\max }, r_{\max }$ are three positive real constants and assumed to be large enough.

Recently, the author in [11] generalized the numerical grid of nodes proposed previously in [15] to tackle (5). However, the investigated numerical method is only of linear convergence rate to estimate the 2 nd derivative terms in (5). Here the aim is to apply the non-uniform computational grid generated in [11], but with higher order FD formulas on these non-equidistant grids so as to keep the second convergence rate along the fully discretized set of equations.

Remark 2.1. A smooth grid mapping affects on truncation errors in Taylor expansions and the arising FD formulas, which make the consistency order of all the approximations in [11, 20] to be at least two, but not the convergence order of these approximations obtained by expanding them via Taylor series.

In practice, the option price is needed at a specific point of the domain, also known as the hot area (specially for the at-the-money case). This hotzone for (5) is a part of the domain where $s, v$ and $r$ tend to the strike price and zero, respectively.

Let $\left\{s_{i}\right\}_{i=1}^{m}$ be a set of non-uniform nodes along $s$ as follows [11]:

$$
s_{i}=\varphi\left(\xi_{i}\right), \quad 1 \leq i \leq m
$$

where $m>1$ and $\xi_{\min }=\xi_{1}<\xi_{2}<\cdots<\xi_{m}=\xi_{\max }$ are $m$ equi-distant points with the following characteristics: $\xi_{\min }=\sinh ^{-1}\left(\frac{s_{\min }-s_{\text {left }}}{d_{1}}\right), \xi_{\text {int }}=\frac{s_{\text {right }}-s_{\text {left }}}{d_{1}}$, $\xi_{\max }=\xi_{\text {int }}+\sinh ^{-1}\left(\frac{s_{\max }-s_{\text {right }}}{d_{1}}\right)$, wherein $s_{\min }=0$. Here $d_{1}>0$ controls the density of the nodes around $s=E$. We also have:

$$
\varphi(\xi)= \begin{cases}s_{\text {left }}+d_{1} \sinh (\xi), & \xi_{\text {min }} \leq \xi<0 \\ s_{\text {left }}+d_{1} \xi, & 0 \leq \xi \leq \xi_{\text {int }}, \\ s_{\text {right }}+d_{1} \sinh \left(\xi-\xi_{\text {int }}\right), & \xi_{\text {int }}<\xi \leq \xi_{\text {max }}\end{cases}
$$

Throughout this work, we used the same value for $d_{1}=\frac{E}{20}$ while $s_{\text {left }}=$ $\max \{0.5, \exp \{-0.25 T\}\} \times E,\left[s_{\text {left }}, s_{\text {right }}\right] \subset\left[0, s_{\max }\right], s_{\text {right }}=E$ and $s_{\max }=$ $14 E$. 
The nodes along $v$, i.e., $\left\{v_{j}\right\}_{j=1}^{n}$ are defined by:

$$
v_{j}=d_{2} \sinh \left(\varsigma_{j}\right), \quad 1 \leq j \leq n,
$$

where $d_{2}>0$ controls the density of the mesh points around $v=0$. Here, we apply $d_{2}=\frac{v_{\max }}{500}$, whereas $v_{\max }=10$. Additionally, here $\varsigma_{j}$ are uniform nodes defined as follows: $\varsigma_{j}=(j-1) \Delta \varsigma, \Delta \varsigma=\frac{1}{n-1} \sinh ^{-1}\left(\frac{v_{\max }}{d_{2}}\right)$, for any $1 \leq j \leq n$. The non-uniform nodes along $r$ are defined as follows:

$$
r_{k}=d_{3} \sinh \left(\zeta_{k}\right), \quad 1 \leq k \leq o,
$$

whereas $d_{3}=\frac{R_{\max }}{500}$ is a positive parameter and $R_{\max }=1$. We also have $\zeta_{k}=(k-1) \Delta \zeta, \Delta \zeta=\frac{1}{o-1} \sinh ^{-1}\left(\frac{r_{\max }}{d_{3}}\right)$. Note that denser mesh points in the important area could circumvent the problems happening in solving (5), like non-smoothness of payoffs (8)-(9) at $s=E$, and the degeneracy at $v=r=0$.

The final non-equidistant computational grid with a focus around the important area is obtained applying a Cartesian product of the grids for $s$ by (15), $v$ by (17) and $r$ by (18) as follows:

Discretized grid $=\left\{s_{i}\right\} \times\left\{v_{j}\right\} \times\left\{r_{k}\right\}, \quad 1 \leq i \leq m, 1 \leq j \leq n, 1 \leq k \leq o$.

\section{Non-uniform discretization}

To attain FD formulations, which are quadratically convergent for all the first and second derivative terms in (5), they should be constructed via interpolating polynomials [8, chapters 2]). The order of the proposed formulas is higher than the existing ones, which are mainly used in the literature related to computational finance and are of second order for the 1st derivative and first order formulas for the 2 nd derivative terms, [15].

To do this analytically, first assume that $f(x)$ is a sufficiently smooth function and $\left\{x_{1}, x_{2}, \cdots, x_{m-1}, x_{m}\right\}$ is a smooth non-uniform grid. To estimate the 1 st derivative by applying three adjacent points $\left\{\left\{x_{i-1}, f\left(x_{i-1}\right)\right\},\left\{x_{i}, f\left(x_{i}\right)\right\}\right.$, $\left.\left\{x_{i+1}, f\left(x_{i+1}\right)\right\}\right\}$, the formula can be written applying a 2 nd order interpolation polynomial going from the three points, as comes next [28]:

$$
f^{\prime}\left(x_{i}\right)=f\left[x_{i-1}, x_{i}\right]-f\left[x_{i+1}, x_{i-1}\right]+f\left[x_{i}, x_{i+1}\right]+\mathcal{O}\left(h^{2}\right),
$$

where $h$ is the maximum local grid spacing. We also have $f\left[x_{l}, x_{q}\right]=\left(f\left(x_{l}\right)-\right.$ $\left.f\left(x_{q}\right)\right) / \Delta x_{l, q}$ with $\Delta x_{l, q}=x_{l}-x_{q}$. Furthermore note that, a FD approximation using $m$ nodes are exact for polynomial functions which are of degree $m-1$. 
In a similar way, we can obtain the quadratic convergence-rate formulas for the points locating on the boundaries as follows:

$$
f^{\prime}\left(x_{1}\right)=f\left[x_{1}, x_{2}\right]+f\left[x_{1}, x_{3}\right]-f\left[x_{3}, x_{2}\right]+\mathcal{O}\left(h^{2}\right),
$$

and

$$
f^{\prime}\left(x_{m}\right)=f\left[x_{m-2}, x_{m}\right]+f\left[x_{m-1}, x_{m}\right]-f\left[x_{m-1}, x_{m-2}\right]+\mathcal{O}\left(h^{2}\right) .
$$

To tackle the cross derivative terms in (5) as elegantly as possible, first differentiation matrices for each dimension is constructed and subsequently the Kronecker product (denoted by $\otimes$ ) of these matrices should be used.

To find the FD formulations possessing quadratic convergence for the second derivative of the function, one more point should be considered. Since the quadratical convergence on non-uniform meshes can be seen once there are four adjacent nodes, i.e., $\left\{\left\{x_{i-2}, f\left(x_{i-2}\right)\right\},\left\{x_{i-1}, f\left(x_{i-1}\right)\right\},\left\{x_{i}, f\left(x_{i}\right)\right\}\right.$, $\left.\left\{x_{i+1}, f\left(x_{i+1}\right)\right\}\right\}$. Thus, the formulas with quadratic convergence is derived by (here $3 \leq i \leq m_{j}-2$ ) as follows:

$$
\begin{aligned}
f^{\prime \prime}\left(x_{i}\right)= & \frac{2\left(\Delta x_{i-1, i}+\Delta x_{i+1, i}\right)}{\Delta x_{i-1, i-2} \Delta x_{i, i-2} \Delta x_{i+1, i-2}} f\left(x_{i-2}\right)+\frac{2\left(\Delta x_{i-2, i}+\Delta x_{i+1, i}\right)}{\Delta x_{i-2, i-1} \Delta x_{i-1, i} \Delta x_{i-1, i+1}} f\left(x_{i-1}\right) \\
& +\frac{2\left(\Delta x_{i-2, i}+\Delta x_{i-1, i}+\Delta x_{i+1, i}\right)}{\Delta x_{i-2, i} \Delta x_{i, i-1} \Delta x_{i, i+1}} f\left(x_{i}\right)+\frac{2\left(\Delta x_{i-2, i}+\Delta x_{i-1, i}\right)}{\Delta x_{i-2, i+1} \Delta x_{i+1, i-1} \Delta x_{i+1, i}} f\left(x_{i+1}\right) \\
& +\mathcal{O}\left(h^{2}\right) .
\end{aligned}
$$

In a similar ways, for the four nodes $\left\{\left\{x_{1}, f\left(x_{1}\right)\right\},\left\{x_{2}, f\left(x_{2}\right)\right\},\left\{x_{3}, f\left(x_{3}\right)\right\}\right.$, $\left.\left\{x_{4}, f\left(x_{4}\right)\right\}\right\}$, we can obtain

$$
\begin{aligned}
f^{\prime \prime}\left(x_{1}\right)= & \frac{2\left(\Delta x_{1,2}+\Delta x_{1,3}+\Delta x_{1,4}\right)}{\Delta x_{1,2} \Delta x_{1,3} \Delta x_{1,4}} f\left(x_{1}\right)+\frac{2\left(\Delta x_{3,1}+\Delta x_{4,1}\right)}{\Delta x_{1,2} \Delta x_{2,3} \Delta x_{2,4}} f\left(x_{2}\right) \\
& +\frac{2\left(\Delta x_{2,1}+\Delta x_{4,1}\right)}{\Delta x_{1,3} \Delta x_{3,2} \Delta x_{3,4}} f\left(x_{3}\right)+\frac{2\left(\Delta x_{2,1}+\Delta x_{3,1}\right)}{\Delta x_{1,4} \Delta x_{4,2} \Delta x_{4,3}} f\left(x_{4}\right)+\mathcal{O}\left(h^{2}\right),
\end{aligned}
$$

and

$$
\begin{aligned}
f^{\prime \prime}\left(x_{2}\right)= & \frac{2\left(\Delta x_{2,3}+\Delta x_{2,4}\right)}{\Delta x_{1,2} \Delta x_{1,3} \Delta x_{1,4}} f\left(x_{1}\right)+\frac{2\left(\Delta x_{1,2}+\Delta x_{3,2}+\Delta x_{4,2}\right)}{\Delta x_{1,2} \Delta x_{2,3} \Delta x_{2,4}} f\left(x_{2}\right) \\
& +\frac{2\left(\Delta x_{1,2}+\Delta x_{4,2}\right)}{\Delta x_{1,3} \Delta x_{3,2} \Delta x_{3,4}} f\left(x_{3}\right)+\frac{2\left(\Delta x_{1,2}+\Delta x_{3,2}\right)}{\Delta x_{1,4} \Delta x_{4,2} \Delta x_{4,3}} f\left(x_{4}\right)+\mathcal{O}\left(h^{2}\right) .
\end{aligned}
$$


Note that $x_{2}$ is not on the boundary but in order to have overall quadratical speed, we require a FD formulation for this node going through $\left\{\left\{x_{1}, f\left(x_{1}\right)\right\}\right.$, $\left.\left\{x_{2}, f\left(x_{2}\right)\right\},\left\{x_{3}, f\left(x_{3}\right)\right\},\left\{x_{4}, f\left(x_{4}\right)\right\}\right\}$.

Similarly, the non-equidistant FD formulas of quadratically convergent for the nodes $\left\{x_{m-1}, x_{m}\right\}$ for estimating the 2 nd derivative can be deduced as comes next:

$$
\begin{aligned}
f^{\prime \prime}\left(x_{m-1}\right)= & \frac{2\left(\Delta x_{m-3, m-1}+\Delta x_{m-2, m-1}\right)}{\Delta x_{m-3, m} \Delta x_{m, m-2} \Delta x_{m, m-1}} f\left(x_{m}\right) \\
& +\frac{2\left(\Delta x_{m-3, m-1}+\Delta x_{m-2, m-1}+\Delta x_{m, m-1}\right)}{\Delta x_{m-3, m-1} \Delta x_{m-1, m-2} \Delta x_{m-1, m}} f\left(x_{m-1}\right) \\
& +\frac{2\left(\Delta x_{m-3, m-1}+\Delta x_{m, m-1}\right)}{\Delta x_{m-3, m-2} \Delta x_{m-2, m-1} \Delta x_{m-2, m}} f\left(x_{m-2}\right) \\
& +\frac{2\left(\Delta x_{m-2, m-1}+\Delta x_{m, m-1}\right)}{\Delta x_{m-2, m-3} \Delta x_{m-1, m-3} \Delta x_{m, m-3}} f\left(x_{m-3}\right)+\mathcal{O}\left(h^{2}\right),
\end{aligned}
$$

and

$$
\begin{aligned}
f^{\prime \prime}\left(x_{m}\right)= & \frac{2\left(\Delta x_{m-3, m}+\Delta x_{m-2, m}+\Delta x_{m-1, m}\right)}{\Delta x_{m-3, m} \Delta x_{m, m-2} \Delta x_{m, m-1}} f\left(x_{m}\right) \\
& +\frac{2\left(\Delta x_{m-3, m}+\Delta x_{m-2, m}\right)}{\Delta x_{m-3, m-1} \Delta x_{m-1, m-2} \Delta x_{m-1, m}} f\left(x_{m-1}\right) \\
& +\frac{2\left(\Delta x_{m-3, m}+\Delta x_{m-1, m}\right)}{\Delta x_{m-3, m-2} \Delta x_{m-2, m-1} \Delta x_{m-2, m}} f\left(x_{m-2}\right) \\
& +\frac{2\left(\Delta x_{m-2, m}+\Delta x_{m-1, m}\right)}{\Delta x_{m-2, m-3} \Delta x_{m-1, m-3} \Delta x_{m, m-3}} f\left(x_{m-3}\right)+\mathcal{O}\left(h^{2}\right) .
\end{aligned}
$$

\section{The semi-discrete system}

One way for imposing the impact of (20)-(27) is with matrices including the weights of (20)-(27), i.e., the non-equidistant second-order FD weights, as their elements. A matrix which shows an estimation to the differential operator is called as a matrix of differentiation [8]. Forming and implementing the proposed scheme based on these matrices are invaluable as aids for analysis.

Hence, the PDE (5) is discretized along the spatial variables, and we can write

$$
\frac{\partial U(t)}{\partial t}=A(t) U(t), \quad 0 \leq t \leq T
$$

at which $U(t)=(\underbrace{u_{1,1,1}(t), u_{1,1,2}(t), \ldots, u_{m, n, o-1}(t), u_{m, n, o}(t)}_{N \text { elements }})^{*}$, is the unknowns vector and $N=m \times n \times o$. The coefficient matrix $A(t)$ is time 
varying because $b$ is time-varying. The semi-discretization matrix $A(t)$ is defined by:

$$
\begin{aligned}
A(t)= & \frac{1}{2} \mathbf{S}^{2} \mathbf{V}\left(D_{s s} \otimes I_{v} \otimes I_{r}\right)+\frac{1}{2} \sigma_{1}^{2} \mathbf{V}\left(I_{s} \otimes D_{v v} \otimes I_{r}\right) \\
& +\frac{1}{2} \sigma_{2}^{2} \mathbf{R}\left(I_{s} \otimes I_{v} \otimes D_{r r}\right)+\rho_{12} \sigma_{1} \mathbf{S V}\left(D_{s} \otimes D_{v} \otimes I_{r}\right) \\
& +\rho_{13} \sigma_{2} \mathbf{S}(\mathbf{V R})^{\frac{1}{2}}\left(D_{s} \otimes I_{v} \otimes D_{r}\right)+\rho_{23} \sigma_{1} \sigma_{2}(\mathbf{V R})^{\frac{1}{2}}\left(I_{s} \otimes D_{v} \otimes D_{r}\right) \\
& +\mathbf{R S}\left(D_{s} \otimes I_{v} \otimes I_{r}\right)+\kappa(\eta I-\mathbf{V})\left(I_{s} \otimes D_{v} \otimes I_{r}\right) \\
& +a(b(T-t) I-\mathbf{R})\left(I_{s} \otimes I_{v} \otimes D_{r}\right)-r I
\end{aligned}
$$

wherein

$$
I=I_{s} \otimes I_{v} \otimes I_{r}
$$

is an identity matrix of the size $N \times N, I_{s}$ is the unit matrix of the size $m \times m$ along $s$, and similarly for $I_{v}$ and $I_{r}$. The other identity matrices are defined similarly. The square matrices $D_{s}, D_{v}, D_{r}, D_{s s}, D_{v v}, D_{r r}$ are sparse differentiation matrices built via the non-equidistant weighting coefficients in Section 3. The diagonal matrices $\mathbf{S}, \mathbf{V}$ and $\mathbf{R}$ are defined as:

$$
\begin{aligned}
& \mathbf{S}=\operatorname{diag}\left(s_{1}, s_{2}, \cdots, s_{m}\right) \otimes I_{v} \otimes I_{r}, \\
& \mathbf{V}=I_{s} \otimes \operatorname{diag}\left(v_{1}, v_{2}, \cdots, v_{n}\right) \otimes I_{r}, \\
& \mathbf{R}=I_{s} \otimes I_{v} \otimes \operatorname{diag}\left(r_{1}, r_{2}, \cdots, r_{o}\right) .
\end{aligned}
$$

All the FD formulas used to construct $A(t)$ are of second-order accuracy in space.

Remark 4.1. One novelty of this work in this section was to first find the weighting coefficients for the fully second order non-uniform (adaptive) grid of discretization points and second to gather all the weights into differentiation matrices to come up with sparse matrices which are much useful in calculations (as will be observed in the next subsection) in terms of computational speed-up.

\subsection{Boundary treatment}

As of yet, the matrix $A_{N \times N}(t)$ has been constructed without incorporating the side conditions. The boundary condition is a set of constraints that define the behavior of unknown solution on the spatial boundary of the domain, [9]. 
By incorporating these conditions (see for instance [27]), we have the following set of linear homogenous ODEs for our new method:

$$
\begin{aligned}
& \frac{\partial U(t)}{\partial t}=\bar{A}(t) U(t)=G(t, U(t)), \quad 0 \leq t \leq T, \\
& U(s, v, r, 0)=(8) \text { or }(9),
\end{aligned}
$$

wherein $\bar{A}(t)$ is the system matrix with the boundaries involved.

\section{Temporal discretization}

The system matrix $\bar{A}(t)$ in (34) is time varying and continuous on some $I \subseteq \mathbb{R}$. Since the IVP (34) homogenous and reads the criterion of the existence and uniqueness theorem [23], viz, we obtain

$$
|\bar{A}(t) U(t)| \leq \| \bar{A}(t)|||U(t)| \leq \mathcal{L}|U(t)|, \quad \mathcal{L}>0,
$$

we can mention that if the matrix function $\bar{A}(t)$ be continuous on some interval $I \subseteq \mathbb{R}$, then due to the theory of matrix functions [13], the solution extends to the whole working interval. Noting that it is necessary that $\bar{A}(t)$ has boundedness or has monotonicity/positivity property.

For linear homogeneous system (34), a naïve solution under several strict conditions $[25$, Chapter 6$]$ is written as comes next:

$$
U(t)=\exp \left(\int_{0}^{t} \bar{A}(\tau) d \tau\right) U(0)
$$

Noting that based on principle of superposition [23], if $x_{1}, x_{2}$ solve (34) then their linear combination $\alpha_{1} x_{1}+\alpha_{2} x_{2}$ also solves (34). Although the solution of the problem (34) could be obtained via (36) under several strict conditions, the computation of one Cauchy integral and a matrix exponential function which is based on a very large banded matrix makes us to depend on time discretization by computational time-stepping solvers.

Assume that $\mathbf{u}_{\iota}$ is a numerical solution to the true solution $\mathbf{u}\left(t_{\iota}\right)$. By considering $\varpi+1$ temporal nodes, a temporal step size $\Delta t=\frac{T}{\varpi}>0$, we consider:

$$
t_{\iota+1}=t_{\iota}+\Delta t, \quad 0 \leq \iota \leq \varpi,
$$

and $\mathbf{u}_{0}=u(s, v, r, 0)$. Then, second-order explicit Runge-Kutta method [3, page 95], which is a member of the Runge-Kutta iterations, can be given as follows:

$$
\mathbf{u}_{\iota+1}=\mathbf{u}_{\iota}+\psi_{2}+\mathcal{O}\left(\Delta t^{3}\right)
$$


wherein

$$
\psi_{2}=\Delta t G\left(t_{\iota}+\frac{1}{2} \Delta t, \mathbf{u}_{\iota}+\frac{1}{2} \psi_{1}\right)
$$

and

$$
\psi_{1}=\Delta t G\left(t_{\iota}, \mathbf{u}_{\iota}\right)
$$

Remark 5.1. The motivation for selecting (38) is to obtain a general quadratical convergence for the fully discretized system of equation. This order of accuracy is consistent with the order of accuracy for spatial discretization discussed in Section 3. Furthermore, the calculation of the derivatives for the time-stepping solver is not required in this case.

\subsection{A stability bound}

In the sequel, we study that under what criteria the numerical discretized solution does not blow up. The following theorem is one of the contributions of this work. This is given for the time-independent case, i.e., when $\bar{A}(t)=\bar{A}$.

Theorem 5.1. Let the function $G(t, U)=\bar{A} U(t)$ satisfies (35). Then, the presented numerical method to option price (5) when $b$ is constant, $h \rightarrow 0$, by (34)E(38) is A-stable conditionally.

Proof 5.1. The method (38) to solve (34) can also be furnished as follows:

$$
\mathbf{u}_{\iota+1}=\left(I+\Delta t \bar{A}+\frac{(\Delta t \bar{A})^{2}}{2}\right) \mathbf{u}_{\iota}
$$

Accordingly, (41) is stable as long as the eigenvalues of matrix $\left(I+\Delta t \bar{A}+\frac{(\Delta t \bar{A})^{2}}{2}\right)$ have modulus less or equal than one. Thus, we can write

$$
1+\Delta t \omega_{\mathfrak{i}}+\frac{\left(\Delta t \omega_{\mathfrak{i}}\right)^{2}}{2}
$$

where $\omega_{\mathrm{i}}$ are the eigenvalues of matrix $\bar{A}$. Therefore, the time-stability is reduced to

$$
\left|1+\Delta t \omega_{\mathfrak{i}}+\frac{\left(\Delta t \omega_{\mathfrak{i}}\right)^{2}}{2}\right| \leq 1, \quad \mathfrak{i}=1,2, \ldots, N
$$

Taking (43) into consideration, the following bounds for the real and imaginary parts of the eigenvalues can be obtained

$$
-\frac{2}{\Delta t} \leq R e\left(\omega_{\mathfrak{i}}\right) \leq 0,
$$


and

$$
-\Pi_{\mathfrak{i}} \leq \operatorname{Im}\left(\omega_{\mathfrak{i}}\right) \leq \Pi_{\mathfrak{i}},
$$

where $\operatorname{Re}(\cdot)$ and $\operatorname{Im}(\cdot)$ are the real and imaginary parts, while

$$
\Pi_{\mathfrak{i}}=\sqrt{2 \sqrt{-\frac{R e\left(\omega_{\mathfrak{i}}\right)\left(\Delta t \operatorname{Re}\left(\omega_{\mathfrak{i}}\right)+2\right)}{\Delta t^{3}}}-\frac{\operatorname{Re}\left(\omega_{\mathfrak{i}}\right)\left(\Delta t R e\left(\omega_{\mathfrak{i}}\right)+2\right)}{\Delta t}} .
$$

Therefore, the proposed procedure is stable as long as the step size $\Delta t$ satisfy (44) and (45) for any eigenvalues of $\bar{A}$. Accordingly, a time-stability bound can be derived by

$$
0<\Delta t \leq \frac{-2}{\rho(\bar{A})}
$$

where $\rho(\bar{A})$ stands for the spectral radius of $\bar{A}$ (the largest negative eigenvalue of $\bar{A})$. Once needed, this spectral radius can be calculated by finding the largest eigenvalue of $\bar{A}$. As an illustration, this can be pursued real quick in Mathematica programming language [30] via the command Eigenvalues[matrix, 1]. The proof is now complete.

Remark 5.2. At the first sight, (47) is not given in terms of the spatial step size (viz, $h$ as the maximum of the non-uniform step size discretization). In fact, $h$ is clearly affect the entries of the matrix $\bar{A}$ and subsequently its eigenvalues. Due to the $3 D$ nature of the model, high size of $\bar{A}$ as well as incorporating the boundary conditions directly into the system matrix rows, the bound (47) sounds to be a practical way for finding a stability bound.

\subsection{Convergence study of the new scheme}

An FD method for numerically solving a PDE problem (5) is convergent, if for any solution to the PDE, $u(s, v, r, t)$, and solutions to the FD procedure such that $\mathbf{u}\left(t_{0}\right)$ be the initial condition, then it converges to $u(s, v, r, t)$ as $\Delta t, h \rightarrow 0$.

Given a PDE of the form $P u=f$ and a FD method, $P_{\Delta t, h} U=f$, it is stated that the FD method is consistent with the PDE problem if for any smooth function $\phi(s, v, r, t)$, the following holds:

$$
P \phi-P_{\Delta t, h} \phi \rightarrow 0, \quad \Delta t, h \rightarrow 0 .
$$

In the stability region, the proposed FD numerical scheme is consistent with the well-posed linear PDE (5). Also, let that the global error at time $T=\varpi \Delta t$ is a grid function given as follows:

$$
E_{\varpi}=Q_{\varpi}-q_{\varpi}, \quad \forall \iota \leq \varpi,
$$


wherein $Q_{\iota}$ is the computational estimation and $q_{\iota}$ is the exact solution. The numerical scheme is shown by $\mathcal{N}(\cdot)$, such that:

$$
Q_{\iota+1}=\mathcal{N}\left(Q_{\iota}\right)
$$

Under the stability condition for the proposed FD scheme $\mathcal{N}$ and its linearity feature along with the fact that $\bar{A}(t)$ has boundedness (the solution is also not oscillatory in the stability region and ignoring the round-off errors, although a drift term exist), there is a constant $\chi$ such that we have:

$$
\left\|\mathcal{N}_{\iota}\right\| \leq \chi, \quad \forall \iota \leq \varpi=T / \Delta t .
$$

On the other hand, the local truncation error can be defined as comes next:

$$
\tau_{\iota}=\frac{1}{\Delta t}\left[\mathcal{N}\left(q_{\iota}\right)-q_{\iota+1}\right]
$$

The consistency means that $\tau_{\iota} \rightarrow 0$, as $\Delta t \rightarrow 0$. To show that how the method converges, now we proceed as follows:

$$
\begin{aligned}
E_{\iota+1} & =Q_{\iota+1}-q_{\iota+1} \\
& =\mathcal{N}\left(Q_{\iota}\right)-q_{\iota+1} \\
& =\mathcal{N}\left(E_{\iota}+q_{\iota}\right)-q_{\iota+1} \\
& =\mathcal{N}\left(E_{\iota}+q_{\iota}\right)-\mathcal{N}\left(q_{\iota}\right)+\mathcal{N}\left(q_{\iota}\right)-q_{\iota+1} \\
& =\mathcal{N}\left(E_{\iota}+q_{\iota}\right)-\mathcal{N}\left(q_{\iota}\right)+\Delta t \tau_{\iota} \\
& =\mathcal{N}\left(E_{\iota}\right)+\Delta t \tau_{\iota} .
\end{aligned}
$$

In fact, it is proved that $\left\|E_{\varpi}\right\| \rightarrow 0$, as $\Delta t, h \rightarrow 0$. Using (53), it is possible to observe that the needs for convergence are the stability and the consistency of the proposed computational scheme. Note that the proposed approach is of quadratical speed, viz, $\mathcal{O}\left(h^{2}\right)+\mathcal{O}\left(\Delta t^{2}\right)$ under the stability condition.

\section{Financial experiments}

In this section, a number of tests are provided in case of at-the-money options. We also compare the results with the uniform FD scheme [29], which is via 2nd-order FD formulations and Euler's time-stepping scheme (denoted by FD), and the non-equally-spaced scheme (via Douglas method) discussed in [11], (denoted by TM). The presented procedure (34)\&(38) is denoted by PM. The following remarks are in order:

- The specifications of our machine are 16.00 GB of RAM and Windows 7 with Intel(R) Core(TM) i5-2430M CPU 2.40GHz processor. 

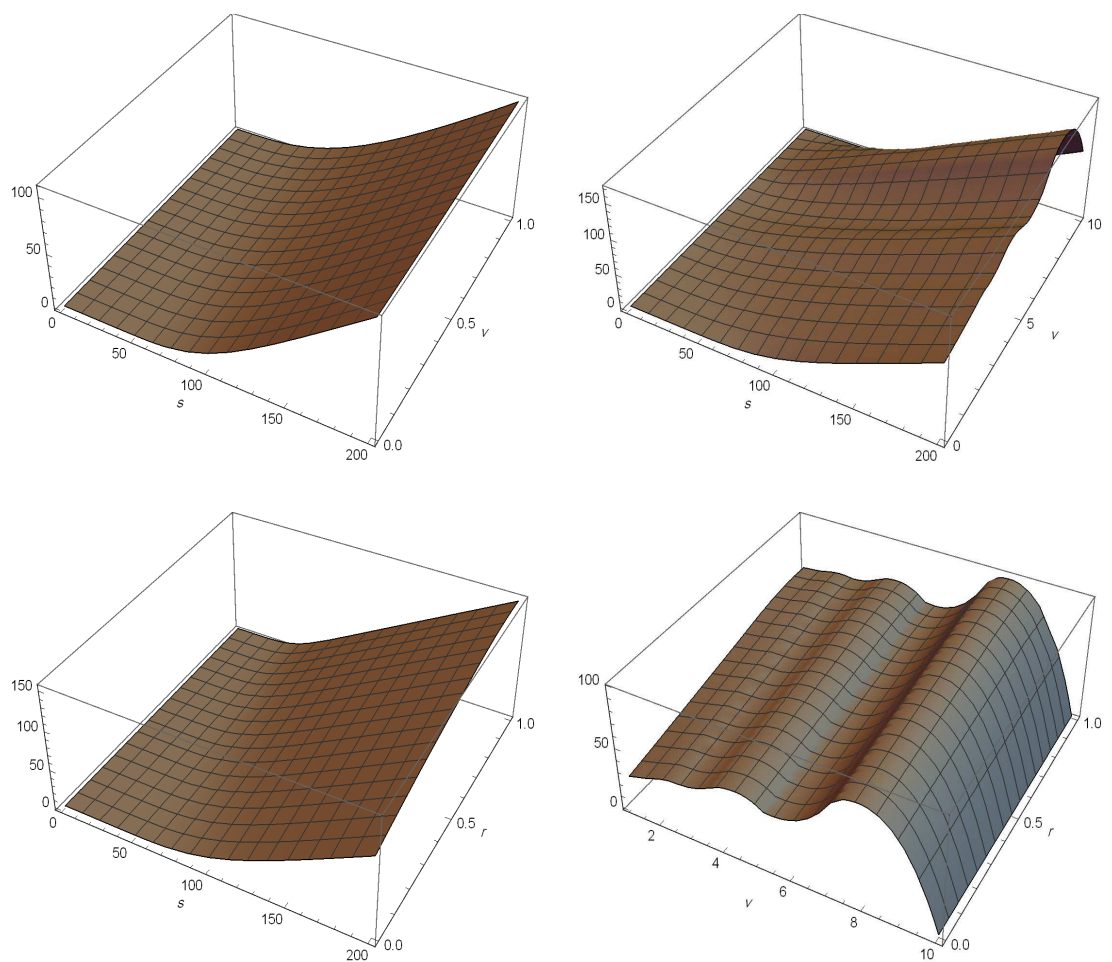

Figure 1: The computed solution in Case I for $u(s, v, 0.024,1)$ when $0 \leq s \leq 200$, $0 \leq v \leq 1$ (top-left), for $u(s, v, 0.024,1)$ when $0 \leq s \leq 200,0 \leq v \leq 10$ (topright), for $u(s, 0.04, r, 1)$ when $0 \leq s \leq 200,0 \leq r \leq 1$ (bottom-left) and for $u(100, v, r, 1)$ when $1 \leq v \leq 10,0 \leq r \leq 1$ (bottom-right).

- The code all the compared method in Mathematica 11.0 (one may refer to [31, Chapter 14] to have some background).

- Computational time is shown by time in seconds.

- More importantly, all the compared schemes have been implemented in a same environment so as to have a fair comparison.

We use the following criterion to check the convergence of the schemes

$$
\text { Error }=\left|\frac{u_{\text {approx }}(s, v, r, t)-u_{\text {ref }}(s, v, r, t)}{u_{\text {ref }}(s, v, r, t)}\right|,
$$

wherein $u_{\text {ref }}$ and $u_{\text {approx }}$ are the exact and numerical results. 


\begin{tabular}{|c|c|c|c|}
\hline & Case I & Case II & Case III \\
\hline$T$ & 1 & 1 & 0.25 \\
$E$ & 100 & 100 & 100 \\
$\kappa$ & 3.0 & 0.3 & 1.0 \\
$\eta$ & 0.12 & 0.04 & 0.09 \\
$\sigma_{1}$ & 0.04 & 0.90 & 1.00 \\
$a$ & 0.20 & 0.16 & 0.22 \\
$\sigma_{2}$ & 0.03 & 0.03 & 0.11 \\
$\rho_{12}$ & 0.6 & -0.5 & -0.3 \\
$\rho_{13}$ & 0.2 & 0.2 & -0.5 \\
$\rho_{23}$ & 0.4 & 0.1 & -0.2 \\
$c_{1}$ & 0.05 & 0.055 & 0.034 \\
$c_{2}$ & 0 & 0 & 0.014 \\
$c_{3}$ & 0 & 0 & 2.10 \\
\hline
\end{tabular}

Table 1: Parameter settings for the model (5).

As suggested in [30, Chapter 1.11], to increase the computational efficiency for very large scale semi-discrete systems that we are dealing with, here we set AccuracyGoal $\rightarrow$ 5, PrecisionGoal $\rightarrow 5$.

Here, we consider more number of discretization nodes along $s$ rather than $v$ and $r$, since its working interval is larger than the others and the non-smoothness of the initial condition occurs along this spatial variable.

Various reports are given in Table 1 (using [11]). The non-constant $b$ is defined as follows:

$$
b(\tau)=c_{1}-c_{2} \exp \left(-c_{3} \tau\right), \quad \tau \geq 0,
$$

where $c_{1}, c_{2}, c_{3}$ are constants, and $\tau=T-t$.

Remark 6.1. Throughout this section, the timing reports as basis for methods' performance comparison make sense, since the timing results are reported based on Mathematica codes which have been written in a same and fair environment and commands using Mathematica 11.0 in a same office laptop. That is to say, all the implementations for the compared methods have been written in a same environment via similar commands.

Table 2 reveals a comparison among the available schemes and the presented procedure. It shows that PM is better than the FD and the TM schemes.

The reference price in Case $\mathrm{I}$ is taken into account via a refined mesh grid as follows: $u(100,0.04,0.024,1) \simeq 13.444$. Table 1 too show that the proposed procedure is efficient. 
ON AN IMPROVED COMPUTATIONAL SOLUTION FOR

\begin{tabular}{|c|c|c|c|c|c|c|c|c|}
\hline Procedure & $m$ & $n$ & $o$ & Size & $\Delta t$ & Price & Error & Time \\
\hline FD & & & & & & & & \\
\hline & 10 & 10 & 10 & 1000 & 0.001 & 21.187 & $5.75 \times 10^{-1}$ & 0.52 \\
& 16 & 12 & 12 & 2304 & 0.0005 & 5.887 & $5.62 \times 10^{-1}$ & 1.15 \\
& 30 & 16 & 16 & 7680 & 0.0001 & 7.542 & $4.38 \times 10^{-1}$ & 12.16 \\
& 40 & 20 & 20 & 16000 & 0.00005 & 10.698 & $2.04 \times 10^{-1}$ & 54.33 \\
& 54 & 22 & 22 & 26136 & 0.00002 & 10.738 & $2.01 \times 10^{-1}$ & 386.97 \\
\hline TM & & & & & & & & \\
\hline & 10 & 10 & 10 & 1000 & 0.001 & 12.216 & $9.12 \times 10^{-2}$ & 0.71 \\
& 16 & 12 & 12 & 2304 & 0.0005 & 13.046 & $2.95 \times 10^{-2}$ & 1.97 \\
& 30 & 16 & 16 & 7680 & 0.0001 & 13.325 & $8.82 \times 10^{-3}$ & 16.60 \\
& 40 & 20 & 20 & 16000 & 0.00005 & 13.376 & $4.99 \times 10^{-3}$ & 101.06 \\
& 54 & 22 & 22 & 26136 & 0.00002 & 13.404 & $2.92 \times 10^{-3}$ & 479.61 \\
\hline PM & & & & & & & & \\
\hline & 10 & 10 & 10 & 1000 & 0.001 & 14.944 & $1.11 \times 10^{-1}$ & 0.66 \\
& 16 & 12 & 12 & 2304 & 0.0005 & 13.804 & $2.68 \times 10^{-2}$ & 1.74 \\
& 30 & 16 & 16 & 7680 & 0.0001 & 13.515 & $5.28 \times 10^{-3}$ & 22.98 \\
& 40 & 20 & 20 & 16000 & 0.00005 & 13.477 & $2.49 \times 10^{-3}$ & 109.22 \\
& 54 & 22 & 22 & 26136 & 0.00002 & 13.457 & $9.87 \times 10^{-4}$ & 505.03 \\
\hline
\end{tabular}

Table 2: Convergence history for various methods in Case I.

To check the smoothness of the computational results of PM, we illustrate the graph of the computational solutions in Figure 1 for various kinds of domains. Figure 1 reveal that the new method has stability by keeping the positivity.

Figure 2 illustrates the stability and positivity of the computational solution in two-dimensional graphs as well as the numerical approximation of the Greeks which show a clear positivity of these important measures by the proposed scheme for dealing with this challenging $3 \mathrm{D}$ problem. In Figure 3 (top), the sparsity pattern of the coefficient matrix $A(t)$ in Case I is brought forward for the methods TM and PM which show a banded matrix.

The results for Case II are shown in Table 3, using various number of discretization points. Similarly the reference price is taken into account at the hot zone $u(100,0.04,0.024,1) \simeq 6.839$. The importance of Case II is that it leads to Greeks which are clearly non-smooth at the strike price $s=E$ and due to this, it is important to compute the numerical solution and then the Greeks as efficiently and accurately as possible. In Figure 3 (bottom), the Greeks for the Case II are given based on PM and show a clear stable and positive behavior for the Greeks, while there is a clear non-differentiability at the strike price. In the meantime, (5) does not satisfy the Feller condition (6) for Case II. Although the standard FD method has an error of magnitude 2, the point is, it does not preserve the positivity of the numerical solution as can be observed in Figure 4 . 

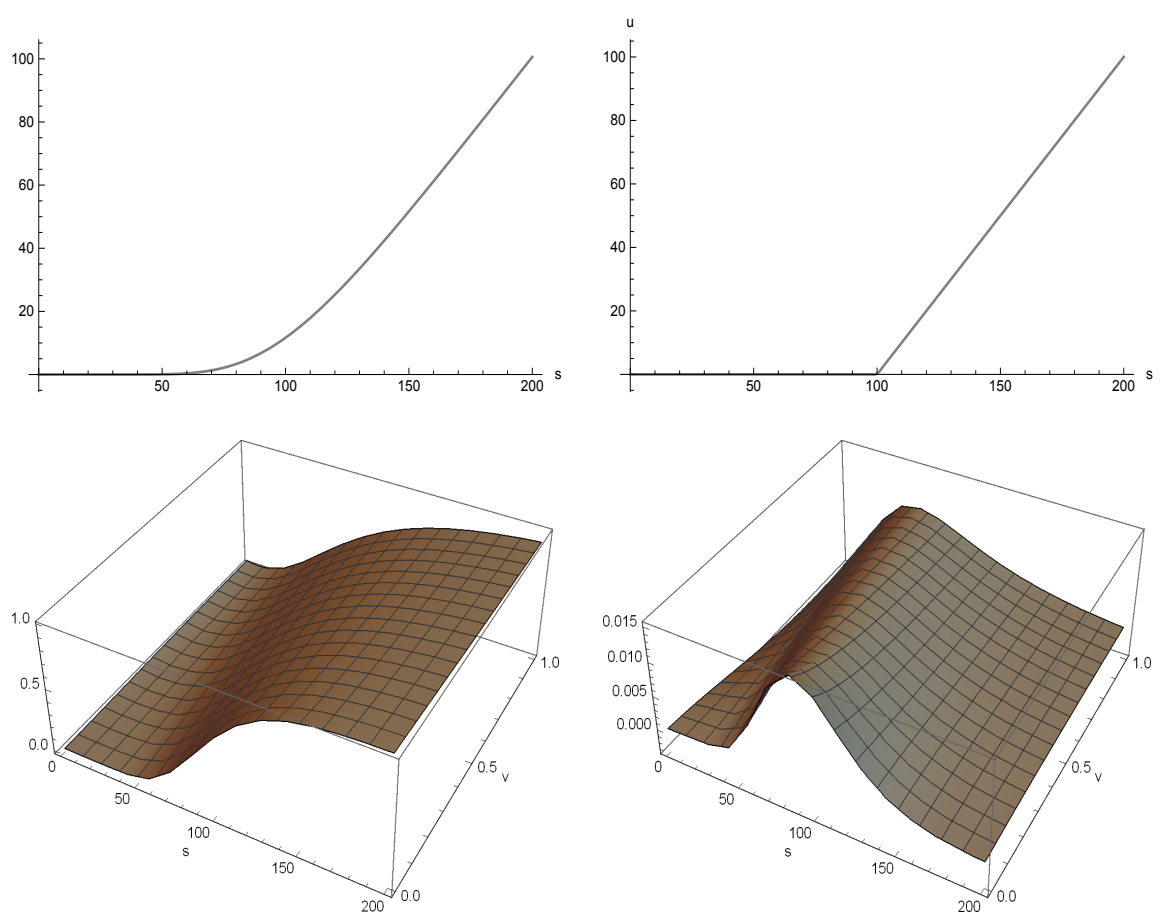

Figure 2: The computed solution in Case I for $u(s, 0,0,1)$ (top-left), for $u(s, 10,1,1)$ (top-right), the Greek delta (bottom-left) and Greek gamma (bottom-right) at $r=0.024$.

Remark 6.2. An explicit method, such as (38), might have trouble when higher accuracy is requested (by increasing the number of nodes for discretizing space), as the time stepsize needs to be particularly small. However, the stability bound (47) can quickly give a good choice for $\Delta t$, which is not that fine to be time-consuming as well.

Remark 6.3. The results presented here are for coarse and fine grid sizes and temporal discretizations, but not as fine as the grid sizes given in [11]. This is to show that the proposed method converge really faster than the TM, and accordingly it is not required to refine the spatial domain more. The numerical results are accurate in the absolute error sense up to $10^{-4}$.

To numerically observe an estimate of the computational convergence speed, we apply the following quotient formula to give a ratio once we double the 

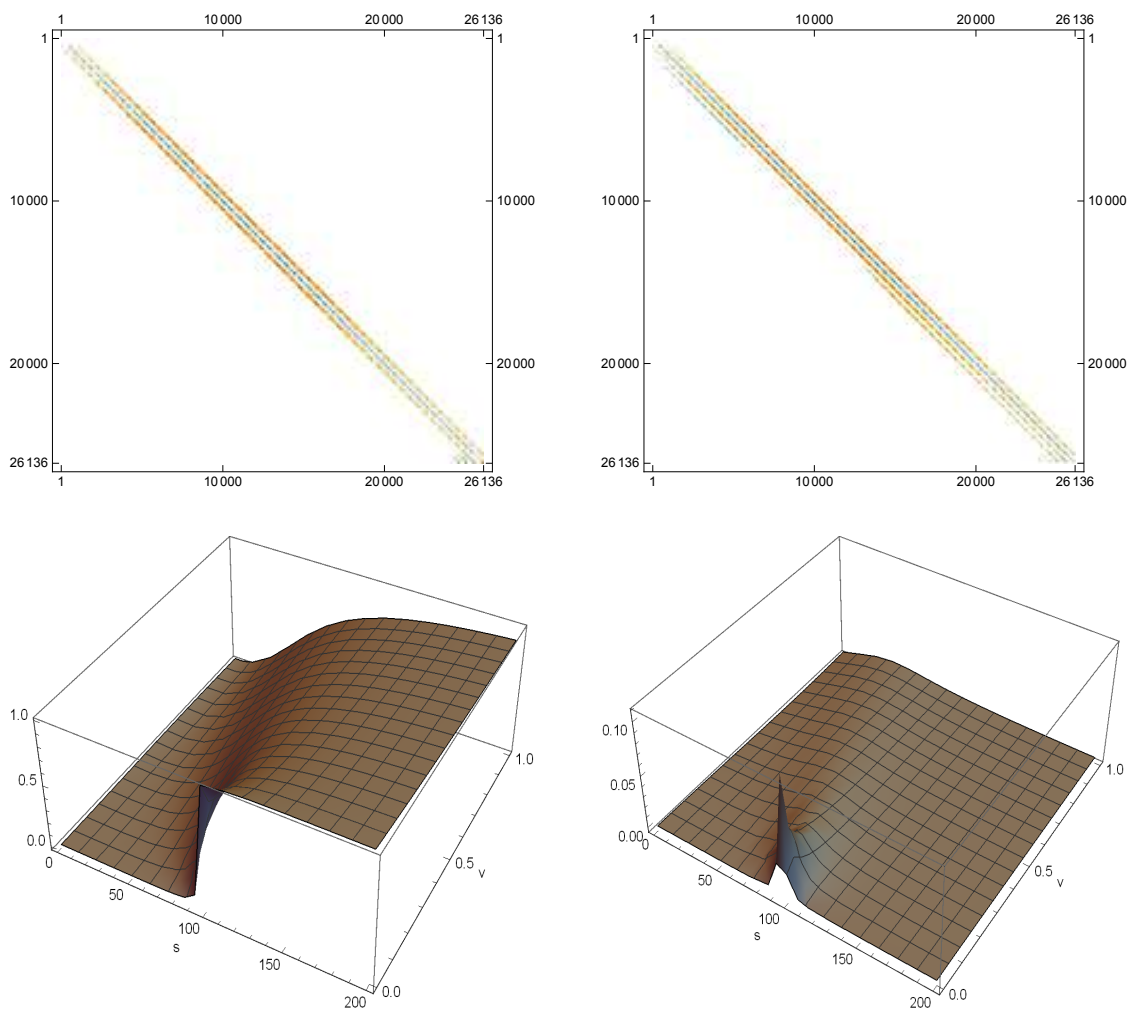

Figure 3: In Case I: the sparsity pattern for the TM (top-left) and for the PM (top-right) when $m=54, n=22, o=22$. In Case II: the Greek delta (bottom-left) and Greek gamma (bottom-right) at $r=0.024$.

number of (non-uniform) grid points for each dimension):

$$
\mathrm{COC}_{(m, n, o)} \simeq\left|\log _{2} \frac{u_{\text {approx }}(2 m, 2 n, 2 o)-u_{\text {approx }}(m, n, o)}{u_{\text {approx }}(m, n, o)-u_{\text {approx }}(m / 2, n / 2, o / 2)}\right|,
$$

and $\mathbf{C O C}$ as the mean of $\mathrm{COC}_{(m, n, o)}$. This ratio is given in Table 4 , which confirms a roughly quadratic convergence speed.

In the comparisons Case III is significant, because it yields to a timedependent $A(t)$, which is harder to tackle with using methods for advancing along time. It is noticed that the time stepping method is explicit, no linear system needs to be solved, thus the computation per time-step is proportional to the number of unknowns. 

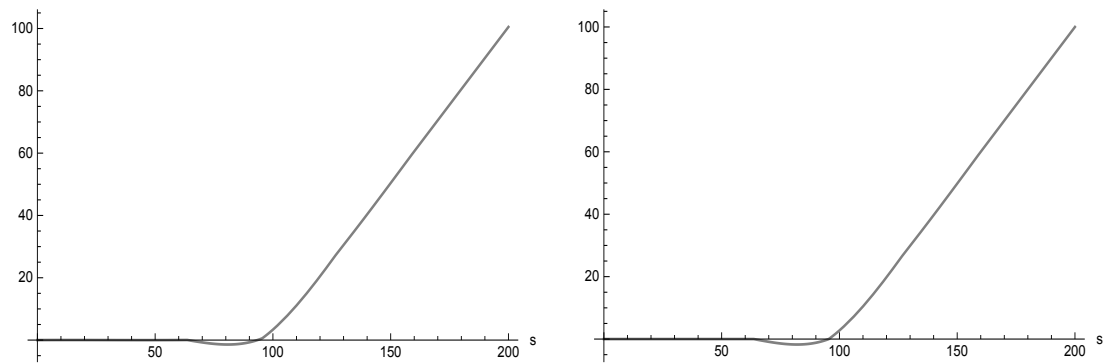

Figure 4: Numerical solution in Case II using FD for $u(s, 0,0,1)$ (left), for $u(s, 10,1,1)$ (right), which reveals that the FD scheme does not preserve the positivity $(m=45, n=24, o=20)$.

\begin{tabular}{|c|c|c|c|c|c|c|c|c|}
\hline Procedure & $m$ & $n$ & $o$ & Size & $\Delta t$ & Price & Error & Time \\
\hline FD & & & & & & & & \\
\hline & 8 & 8 & 8 & 512 & 0.002 & 47.829 & $5.99 \times 10^{0}$ & 0.29 \\
& 14 & 10 & 10 & 1400 & 0.0005 & 5.469 & $2.00 \times 10^{-1}$ & 0.78 \\
& 20 & 14 & 12 & 3360 & 0.00025 & 14.786 & $1.16 \times 10^{0}$ & 2.30 \\
& 24 & 16 & 14 & 5376 & 0.0001 & 12.960 & $8.95 \times 10^{-1}$ & 7.78 \\
& 32 & 18 & 18 & 10368 & 0.00005 & 8.599 & $2.57 \times 10^{-1}$ & 30.26 \\
& 45 & 24 & 20 & 19800 & 0.000025 & 6.456 & $5.59 \times 10^{-2}$ & 182.07 \\
\hline TM & & & & & & & & \\
\hline & 8 & 8 & 8 & 512 & 0.002 & 5.010 & $2.67 \times 10^{-1}$ & 0.31 \\
& 14 & 10 & 10 & 1400 & 0.0005 & 6.440 & $5.83 \times 10^{-2}$ & 0.74 \\
& 20 & 14 & 12 & 3360 & 0.00025 & 6.672 & $2.43 \times 10^{-2}$ & 2.07 \\
& 24 & 16 & 14 & 5376 & 0.0001 & 6.729 & $1.59 \times 10^{-2}$ & 7.68 \\
& 32 & 18 & 18 & 10368 & 0.00005 & 6.797 & $6.02 \times 10^{-3}$ & 35.52 \\
& 45 & 24 & 20 & 19800 & 0.000025 & 6.830 & $1.28 \times 10^{-3}$ & 188.33 \\
\hline PM & & & & & & & & \\
\hline & 8 & 8 & 8 & 512 & 0.002 & 5.794 & $1.52 \times 10^{-1}$ & 0.37 \\
& 14 & 10 & 10 & 1400 & 0.0005 & 6.628 & $3.07 \times 10^{-2}$ & 1.08 \\
& 20 & 14 & 12 & 3360 & 0.00025 & 6.759 & $1.16 \times 10^{-2}$ & 3.89 \\
& 24 & 16 & 14 & 5376 & 0.0001 & 6.776 & $9.07 \times 10^{-3}$ & 14.49 \\
& 32 & 18 & 18 & 10368 & 0.00005 & 6.809 & $4.30 \times 10^{-3}$ & 60.28 \\
& 45 & 24 & 20 & 19800 & 0.000025 & 6.833 & $8.52 \times 10^{-4}$ & 241.54 \\
\hline
\end{tabular}

Table 3: Comparison reports in the Case II.

In Figure 5, we have plotted the numerical solution for our scheme in different positions of the domain. The results show a stable and positive computed solution. Figure 6 (left) is brought forward to manifest the sparsity pattern of our scheme, at which the dark red color is dedicated to the positions, at which $b$ is present as a function of $t$. Figure 6 (right) is given to show that our scheme is convergent by increasing the number of points at the hot zone 


\begin{tabular}{|c|c|c|c|}
\hline$m, n, o$ & Price & COC & COC \\
\hline 4 & 7.9106323130 & - & \\
8 & 5.7940541174 & - & \\
16 & 6.7602509210 & 1.13 & \\
32 & 6.8300971620 & 3.79 & $\mathbf{2 . 4}$ \\
\hline
\end{tabular}

Table 4: Mean of convergence ratio for PM in Case II using a fixed time step-size $\Delta t=0.00002$.

(100, 0.04, 0.024, 0.25), considering:

$h_{\max }=\left(\max \left\{\right.\right.$ differences $\left.\left\{s_{i}\right\}\right\}+\max \left\{\right.$ differences $\left.\left\{v_{j}\right\}\right\}+\max \left\{\right.$ differences $\left.\left.\left\{r_{k}\right\}\right\}\right) / 3$.
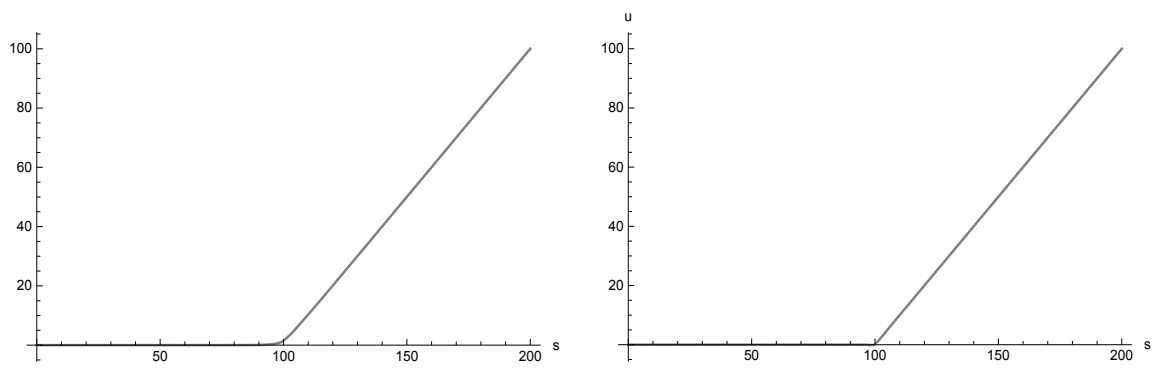

Figure 5: Computational solution in Case III using PM for $u(s, 0,0,0.25)$ (left), for $u(s, 10,1,0.25)$ (right), which reveals that the PM method is stable and preserves the positivity $(m=22, n=16, o=16, \Delta t=0.0001)$.

\section{Summary}

The valuation of several practically significant securities, like those which are callable or path-dependent, are subject to interest rates. This made the quant to construct interest rate models with practical applications.

In this paper, we have applied an non-uniform discretization for the spatial variables in the financial 3D Heston-Cox-Ingersoll-Ross PDE. To have a total fast convergence rate, quadratically convergent discretizations were done for all the first and second derivative terms involved in the model. In addition, the model was introduced in a matrix form using differentiation matrices to 

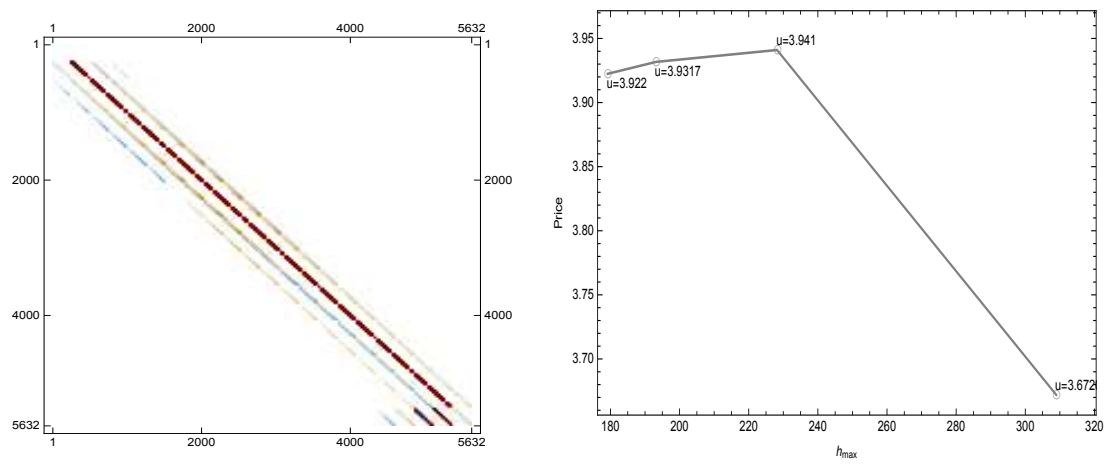

Figure 6: The sparsity pattern of PM for $m=22, n=16, o=16$ (left) and the error decay (right) in Case III.

unify and simplify the procedure while a second-order RK method was applied for time-stepping.

Several financial experiment were discussed and showed that the presented computational method is reliable in terms of being fast and stable for the HCIR PDE and it is therefore useful for situations, at which the Feller condition holds or not and for short and long maturities.

\section{References}

[1] R. Ahlip, M. Rutkowski, Pricing of foreign exchange options under the MPT stochastic volatility model and the CIR interest rates, Euro. J. Finance, 22 (2016), 551-571.

[2] K. Atkinson, W. Han, Theoretical Numerical Analysis, A Functional Analysis Framework, Third Edition, Springer, New York, 2009.

[3] J.C. Butcher, Numerical Methods for Ordinary Differential Equations, 2nd Edition, Wiley, England, 2008.

[4] J.C. Cox, J.E. Ingersoll, S.A. Ross, A theory of the term structure of interest rates, Econometrica, 53 (1985), 385-407.

[5] R. Company, V.N. Egorova, M.El. Fakharany, L. Jódar, F. Soleymani, Numerical Analysis of Novel Finite Difference Methods, Novel Methods in Computational Finance, 2017, 171-214.

[6] E. Ekström, J. Tysk, Boundary conditions for the single-factor term structure equation, Ann. Appl. Prob., 21 (2011), 332-350.

[7] L. Feng, V. Linetsky, Pricing options in jump-diffusion models: An extrapolation approach, Oper. Res., 56 (2008), 304-325. 
[8] B. Fornberg, A Practical Guide to Pseudospectral Methods, Cambridge University Press, UK, 1996.

[9] L.A. Grzelak, C.W. Oosterlee, On the Heston model with stochastic interest rates, SIAM J. Financ. Math., 2 (2011), 255-286.

[10] S. Hadi Seyedi, B. Nemati Saray, A. Ramazani, On the multiscale simulation of squeezing nanofluid flow by a highprecision scheme, Powder Technol., 340 (2018), 264-273.

[11] T. Haentjens, Efficient and stable numerical solution of the Heston-CoxIngersoll-Ross partial differential equation by alternating direction implicit finite difference schemes, Int. J. Comput. Math., 90 (2013), 2409-2430.

[12] S.L. Heston, A closed-form solution for options with stochastic volatility with applications to bond and currency options, Rev. Finan. Stud., 6 (1993), 327-343.

[13] N.J. Higham, Functions of Matrices: Theory and Computation, Society for Industrial and Applied Mathematics, Philadelphia, Pa, USA, 2008.

[14] J.C. Hull, Options, Futures and other Derivatives, 6th ed., Prentice Hall, Upper Saddle River, NJ, 2006.

[15] K.J. in 't Hout, S. Foulon, ADI finite difference schemes for option pricing in the Heston model with correlation, Int. J. Numer. Anal. Model., 7 (2010), 303-320.

[16] A Itkin, New solvable stochastic volatility models for pricing volatility derivatives, Rev. Der. Res., 16 (2013), 111-134.

[17] A. Itkin, Pricing Derivatives Under Lévy Models: Modern FiniteDifference and Pseudo-Differential Operators Approach, Birkhäuser Basel, 2017.

[18] M.K. Kadalbajoo, A. Kumar, L.P. Tripathi, Radial-basis-function-based finite difference operator splitting method for pricing American options, Int. J. Comput. Math., 95 (2018), 2343-2359.

[19] R. Kangro, R. Nicolaides, Far field boundary conditions for Black-Scholes equations, SIAM J. Numer. Anal., 38 (2000), 1357-1368.

[20] T. Kluge, Pricing derivatives in stochastic volatility models using the finite difference method, Dipl. thesis, TU Chemnitz, 2002.

[21] R. Knapp, A method of lines framework in Mathematica, J. Numer. Anal. Indust. Appl. Math. (JNAIAM), 3 (2008), 43-59.

[22] S. Milovanović, L. von Sydow, Radial basis function generated finite differences for option pricing problems, Comput. Math. Appl. 75 (2018), 1462-1481.

[23] A.S. Novozhilov, Lecture Notes, Ordinary Differential Equations I, NonAutonomous Linear Systems of ODE, General theory, USA, pp. 74-86. 
[24] E. Rotenstein, A multi-dimensional FBSDE with quadratic generator and its applications, An. Şt. Univ. Ovidius Constanţa, 23 (2015), 213-222.

[25] W.J. Rugh, Linear System Theory, Second Edition, New Jersey, 1996.

[26] J. Sippel, S. Ohkoshi, All power to PRDC notes, Risk, 15 (2002), 531-533.

[27] M. Sofroniou, R. Knapp, Advanced Numerical Differential Equation Solving in Mathematica, Wolfram Mathematica, Tutorial Collection, USA, 2008.

[28] F. Soleymani, Pricing multi-asset option problems: A Chebyshev pseudospectral method, BIT, 59 (2019), 243-270.

[29] D. Tavella, C. Randall, Pricing Financial Instruments: The Finite Difference Method, New York: John Wiley and Sons, 2007.

[30] M. Trott, The Mathematica Guidebook for Numerics, Springer, New York, NY, USA, 2006.

[31] S. Wagon, Mathematica in Action, Third edition, Springer, New York, 2010.

[32] H. Zhao, X.M. Rong, Y.G. Zhao, Optimal excess-of-loss reinsurance and investment problem for an insurer with jump-diffusion risk process under the Heston model, Insur. Math. Econ., 53 (2013), 504-514.

Fazlollah Soleymani,

Department of Mathematics,

Institute for Advanced Studies in Basic Sciences (IASBS),

Zanjan 45137-66731, Iran.

Emails: fazlollah.soleymani@gmail.com \& soleymani@iasbs.ac.ir

Ali Akgül,

Department of Mathematics,

Art and Science Faculty,

Siirt University, Siirt, Turkey.

Emails: aliakgul@siirt.edu.tr \& aliakgul00727@gmail.com

Esra Karatas Akgül,

Department of Mathematics,

Faculty of Education,

Siirt University, Siirt, Turkey.

Email: esrakaratas@siirt.edu.tr 